\title{
In-situ TEM Study on Sub-10 nm Materials
}

Tao Xu ${ }^{1}$, Yuting Shen ${ }^{1}$, Chao Zhu ${ }^{1}$, Qiubo Zhang ${ }^{1}$, Haimei Zheng ${ }^{2}$, and Litao Sun ${ }^{1}$

1. SEU-FEI Nano-Pico Center, Key Laboratory of MEMS of Ministry of Education, Southeast University, Nanjing, P. R. China

2. Materials Science Division, Lawrence Berkeley National Laboratory, Berkeley, USA

With the development of semiconductor technology, the $10 \mathrm{~nm}$ feature size of fabrication is approaching. It is thus quite essential to explore more precise nanofabrication and characterization method to evaluate the shape/structure stability and possible new properties of sub-10-nm material components, especially under external field such as strain, electric, or thermal fields. Here we review our recent progress in atomic resolution nanofabrication and dynamic characterization of individual nanostructures and nanodevices based on the idea of "setting up a nanolab inside a transmission electron microscope".

Inside the TEM, the electron beam can be used not only to image but also to induce nanofabrication on the atomic scale. Electron beam irradiation can be utilized to etch nanocrystals, as shown in Figure 1, $\mathrm{CaO}$ crystals are etched by an electron beam column by column, and the process is similar to chemical etching [1]. Electron beam irradiation can also be used to repair defects in nanocrystals. Nanopores in $\mathrm{MoS}_{2}$ and $\mathrm{Bi}_{2} \mathrm{Te}_{3}$ can be repaired to high-quality crystals with a low number of defects [2]. Besides, electron beam structuring provides a top-down approach to create quasi-1D structure by cutting $2 \mathrm{D}$ sheets. Robust ultrafine molybdenum-sulfide ribbons with sub-1 nm width can be widely formed in a $\mathrm{MoS}_{2}$ sheet [3]. Single-walled armchair BN tubes can be formed in AA' stacked bilayer h-BN due to the formation of covalent interlayer bonds at two parallel zigzag edges, and the diameter of the tube can be decreased in discrete steps to $0.45 \mathrm{~nm}$, corresponding to a $(3,3)$ tube [4].

Additional probes from a special-designed holder provide the possibility to further manipulate and measure the electric/mechanical properties of the nanostructures in the small specimen chamber of a TEM. As shown in Figure 2, if a $\mathrm{CdS}$ is inserted in the circuit with $\mathrm{Cu}$ electrode, $\mathrm{Cd}$ is eliminated due to Ohmic heating, whereas $\mathrm{Cu}^{+}$migrates into the crystal driven by the electrical field force under electric bias, resulting in the formation of $\mathrm{CdS} @ \mathrm{Cu}_{2} \mathrm{~S}$ core-shell structures [5]. Such an electrically driven cation exchange can be used to grow heterogeneous structures in situ. Some novel phenomena can also be observed if the mechanical force is applied to individual crystal. Sub-10 nm Ag particles can be deformed like a liquid droplet but remain highly crystalline in the interior at room temperature, which is a surface-diffusion-mediated pseudoelastic deformation [6].

Besides, the development of liquid cell TEM technique provides an opportunity to dynamically observe the nucleation and growth of nanocrystals in liquid environment. Figure 3 shows the oriented attachment of Au nanoparticles at $\{111\}$ surface inside aqueous solution [7]. The particle pairs rotate randomly at a separation distance greater than twice the layer thickness of adsorbed ligands. When the particles get closer, the ligands overlap and guide the rotation until the particles share a common $\{111\}$ orientation, then a sudden contact occurs accompanied by the expulsion of the ligands on this surface [8].

\section{References:}

[1] Y. Shen, et al., Nano Letters 17(2017), p.5119. 
[2] Y. Shen, et al., Advanced Materials, DOI: 10.1002/adma.201705954

[3] X. Liu, et al., Nature Communications 4(2013), 1776

[4] T. Xu, et al., Advanced Functional Materials 27(2017),1603897.

[5] Q. Zhang, et al., Nature Communications 8(2017), 14889

[6] J. Sun, et al., Nature Materials 13(2014), p.1007

[7] C. Zhu, et al., Nature Communications 9(2018), 421

[8] The authors acknowledge funding from the National Natural Science Foundation of China (Grant Nos. 51420105003, 11327901, 11525415, and 61601116).

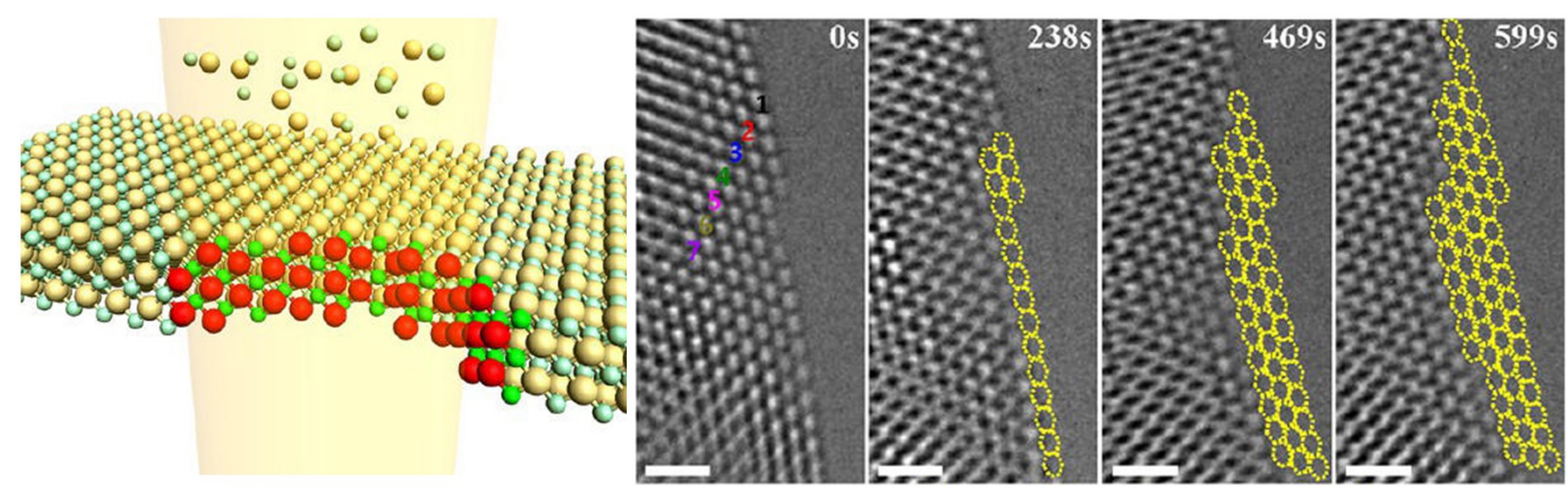

Figure 1. Etching process of $\mathrm{CaO}$ under electron beam. Scale bar is $1 \mathrm{~nm}$

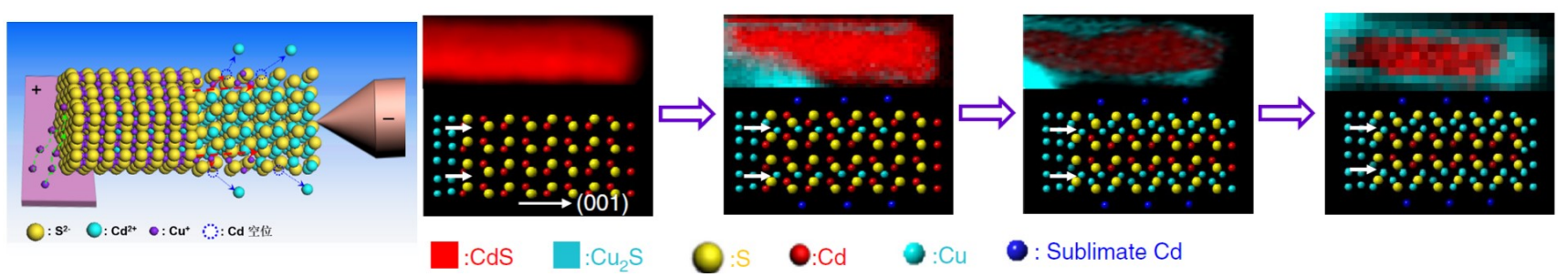

Figure 2. Electrically driven cation exchange for in-situ fabrication of individual nanostructure

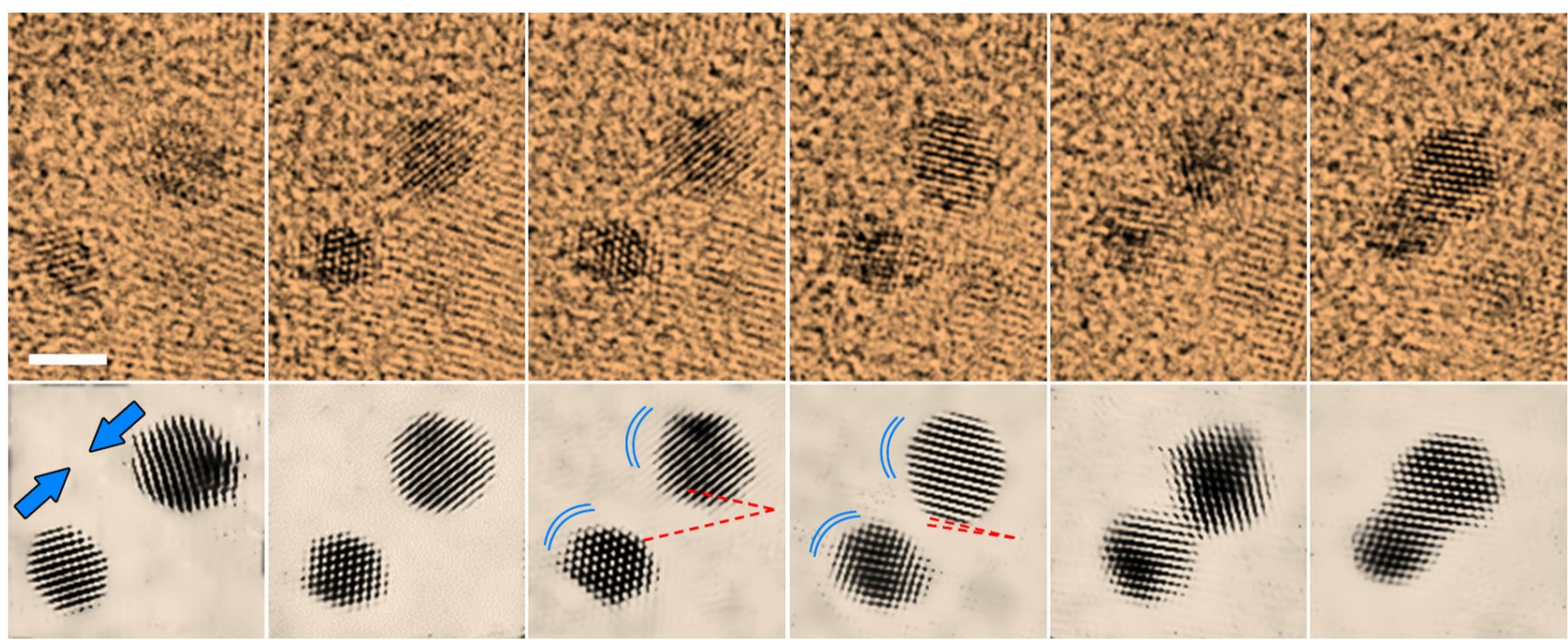

Figure 3. Oriented attachment of $\mathrm{Au}$ nanoparticles at $\{111\}$ surface, evolving into a twin structure. Scale bar is $2 \mathrm{~nm}$ 